\title{
The Relationship Between Fluticasone Furoate Systemic Exposure and Cortisol Suppression
}

\author{
Ann Allen
}

Published online: 30 May 2013

(c) The Author(s) 2013. This article is published with open access at Springerlink.com

\begin{abstract}
Introduction The inhaled corticosteroid (ICS) fluticasone furoate is in development, in combination with the longacting beta ${ }_{2}$-agonist vilanterol for the once-daily treatment of asthma and chronic obstructive pulmonary disease and as a monotherapy treatment for asthma. Corticosteroids, including ICSs, have the potential to induce dose-dependent systemic effects on the hypothalamic-pituitary-adrenal (HPA) axis. Cortisol suppression has been observed in asthma patients with normal HPA axis function at baseline on receiving high doses of ICSs, and is associated with adverse effects on a number of physiological processes. The measurement of 24-h serum cortisol and 24-h urinary cortisol excretion are sensitive methods for assessing adrenocortical activity, and can evaluate cortisol suppression in a dose-dependent manner.

Objective The purpose of the meta-analysis presented here was to characterize the population pharmacokinetic/ pharmacodynamic relationship between fluticasone furoate systemic exposure [as measured by area under the concentration-time curve over $24 \mathrm{~h}$ postdose $\left(\mathrm{AUC}_{24}\right) \mathrm{]}$ and both 24-h weighted mean serum cortisol (WM24) and 24-h urine cortisol excretion in healthy subjects and subjects with asthma.

Methods The serum cortisol meta-analysis integrated eight studies; five Phase I studies in healthy subjects, two Phase IIa studies, and one Phase III study in subjects with asthma. Each study included serial blood sampling for estimation of WM24. The urine cortisol meta-analysis integrated three studies: one Phase I study in healthy
\end{abstract}

\footnotetext{
A. Allen $(\bowtie)$

Clinical Pharmacology Modelling and Simulation,

GlaxoSmithKline R\&D, Stevenage, Hertfordshire SG1 2NY, UK

e-mail: ann.allen@gsk.com
}

subjects, and one Phase IIb and one Phase III study in subjects with asthma. Each study included complete 0-24 h urine collection for estimation of urine cortisol excretion. All studies included blood sampling for estimation of fluticasone furoate $\mathrm{AUC}_{24}$. A sigmoid maximum effect $\left(E_{\max }\right)$ model was fitted to fluticasone furoate $\mathrm{AUC}_{24}$ and serum cortisol and urine cortisol data using nonlinear mixedeffect modeling with the computer program NONMEM ${ }^{\circledR}$. Results Over a wide range of systemic fluticasone furoate exposure representing the therapeutic and supratherapeutic range, the relationship between fluticasone furoate $\mathrm{AUC}_{24}$ and WM24 and 24-h urine cortisol excretion was well described by an $E_{\max }$ model. The average estimate of AUC producing $50 \%$ of maximum effect $\left(\mathrm{AUC}_{50}\right)$ was similar for the serum cortisol and urine cortisol models with values of 1,556 and $1,686 \mathrm{pg} \cdot \mathrm{h} / \mathrm{mL}$, respectively. Although formulation/inhaler was shown to be a significant covariate on the estimates of both WM24 at zero concentration $\left(\mathrm{C}_{0}\right)$ and $\mathrm{AUC}_{50}$ in the serum cortisol model, the differences were small and believed to be due to study variability. Age was shown to be a significant covariate on the estimates of both $C_{0}$ and $\mathrm{AUC}_{50}$ in the urine cortisol model, and was considered to be a reflection of lower urine cortisol excretion in adolescents.

Conclusion A pharmacokinetic/pharmacodynamic model has been established over a wide range of systemic fluticasone furoate exposure representing the therapeutic and supratherapeutic range to both WM24 and 24-h urine cortisol excretion. The values of $\mathrm{AUC}_{50}$ of 1,556 and $1,686 \mathrm{pg} \cdot \mathrm{h} / \mathrm{mL}$, respectively, are several times higher than average fluticasone furoate $\mathrm{AUC}_{24}$ values observed at clinical doses of fluticasone furoate $(\leq 200 \mu \mathrm{g})$. The models predict a fluticasone furoate $\mathrm{AUC}_{24}$ of $1,000 \mathrm{pg} \cdot \mathrm{h} / \mathrm{mL}$ would be required to reduce 24 -h serum cortisol or $24-\mathrm{h}$ urine cortisol excretion by 20 and $17 \%$, respectively. 


\section{Introduction}

The inhaled corticosteroid (ICS) fluticasone furoate (FF; GW685698) is in development, in combination with the long-acting beta $_{2}$-agonist (LABA) vilanterol (VI; GW642444M) for once-daily treatment of asthma and chronic obstructive pulmonary disease (COPD). Fluticasone furoate is also being developed as a monotherapy treatment for asthma. The pharmacokinetic, pharmacodynamic, and safety profiles of the fluticasone furoate/vilanterol combination have been described in healthy subjects as well as in patients with asthma and COPD [1-4]. In addition, once-daily administration of fluticasone furoate/ vilanterol was effective at improving lung function in patients with COPD [5, 6] or asthma [7, 8].

Endogenous cortisol is responsible for several important functions within the body and its level is regulated by a feedback system, involving the hypothalamus, pituitary, and adrenal glands, known as the HPA axis. Corticosteroids, including ICS, have the potential to induce dosedependent systemic effects on the HPA axis [9-11]. High doses of ICSs have resulted in cortisol suppression in asthma patients with normal HPA axis function at baseline $[12,13]$ and this finding is associated with adverse effects on a number of physiological processes [14, 15]. Monitoring systemic cortisol levels is one of the most sensitive markers of HPA suppression and the measurement of 24-h serum cortisol (serum cortisol) is a sensitive method for assessing adrenocortical activity, and can evaluate cortisol suppression in a dose-dependent manner [11]. However, this method requires that serial blood samples are collected over a 24-h period and this is not always feasible in a large clinical trial setting. An acceptable alternative is the use of complete 24-h urine collection for evaluation of cortisol excretion [11].

Although there is extensive data in the literature on other ICSs [11], no data has yet been published for fluticasone furoate characterizing the systemic exposure relationship with reductions in either serum cortisol or urinary cortisol. The purpose of the meta-analysis presented here was to characterize the population pharmacokinetic/pharmacodynamic relationship between fluticasone furoate systemic exposure [as measured by $24-\mathrm{h}$ area under the concentration-time curve $\left.\left(\mathrm{AUC}_{24}\right)\right]$ and 24-h weighted mean serum cortisol (WM24) and also a relationship between fluticasone furoate systemic exposure $\left(\mathrm{AUC}_{24}\right)$ and 24-h urine cortisol excretion in healthy subjects and in subjects with asthma.

The studies included in the meta-analyses allowed investigation of the fluticasone furoate $\mathrm{AUC}_{24}$ versus cortisol relationship over a wide concentration range, as well as assessment of the influence of population (healthy subjects or patients with asthma), formulation/inhaler
[ROTADISK/lactose, DISKUS/cellobiose octaacetate $(\mathrm{COA})+$ lactose or dry powder inhaler (DPI)/lactose] on any pharmacokinetic/pharmacodynamic relationship. ROTADISK and DISKUS (GlaxoSmithKline, UK) included doses up to ten times and eight times greater than the maximum proposed fluticasone furoate therapeutic dose of $200 \mu \mathrm{g}$, whilst for DPI the maximum dose was only four times greater. Most of the repeat dose studies conducted with fluticasone furoate/vilanterol administered via the DPI utilized doses of fluticasone furoate that had no detectable effect on cortisol [16]. In dose ranging studies, fluticasone furoate (doses ranging from 25 to $800 \mu \mathrm{g}$ ) did not significantly suppress 24-h urine cortisol excretion after 8 weeks once-daily dosing relative to placebo, with the exception of the 800- $\mu$ g dose that served to define a supratherapeutic dose [16]. To enable characterization of the relationship between fluticasone furoate $\mathrm{AUC}_{24}$ and cortisol, it was necessary to include studies where supratherapeutic doses up to $2,000 \mu \mathrm{g}$ (representing ten times higher than the maximum clinical dose) were used, which resulted in significant suppression of cortisol production. Many of these studies were conducted in early development and utilized other formulations and/or inhalers.

\section{Methods}

The meta-analysis for the serum cortisol analysis integrated eight studies; five Phase I studies (Studies 1-5) in healthy subjects, two Phase IIa studies in subjects with asthma (Studies 6 and 7), and one Phase III study in subjects with asthma (Study 9). Each of these studies included blood sampling for estimation of $\mathrm{AUC}_{24}$ and serial blood sampling for estimation of WM24 as detailed in Table 1.

The meta-analysis for the urine cortisol analysis integrated three studies: one Phase I study in healthy subjects (Study 5), one Phase IIb study in subjects with asthma (Study 8), and one Phase III study (Study 9). Each of these studies included blood sampling for estimation of fluticasone furoate AUC and complete 24-h urine collection for estimation of 24-h urine cortisol excretion as detailed in Table 1.

The studies used in these meta-analyses used the DPI as well as formulations administered via ROTADISK or DISKUS. It is possible that these different formulations/ inhalers may not have delivered the same lung dose. Because the pharmacokinetic/pharmacodynamic analysis involves the observed systemic exposure, and is related to systemic effects, differences in delivered lung dose have no relevance for these analyses and therefore pooling of data from these studies is valid. 


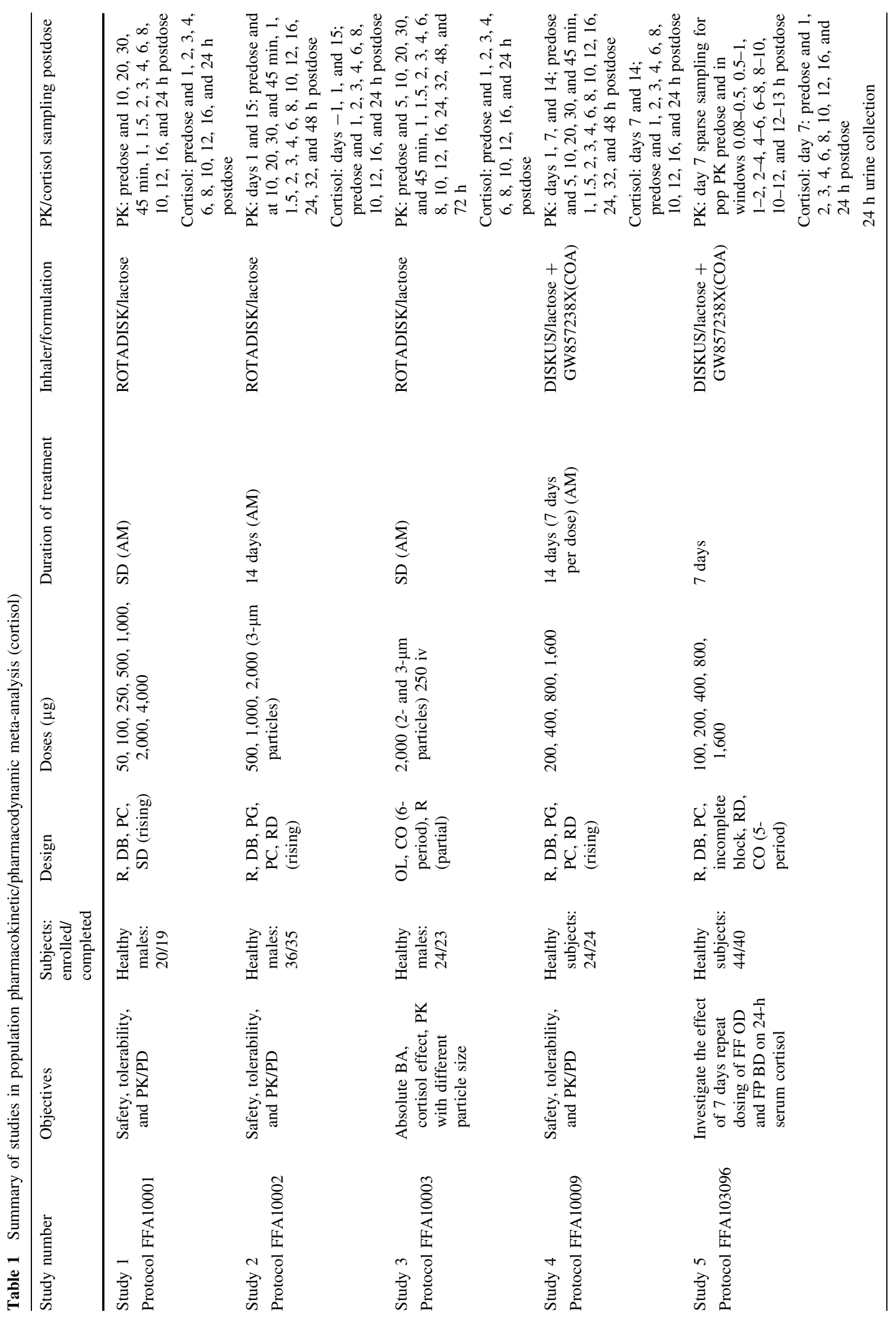




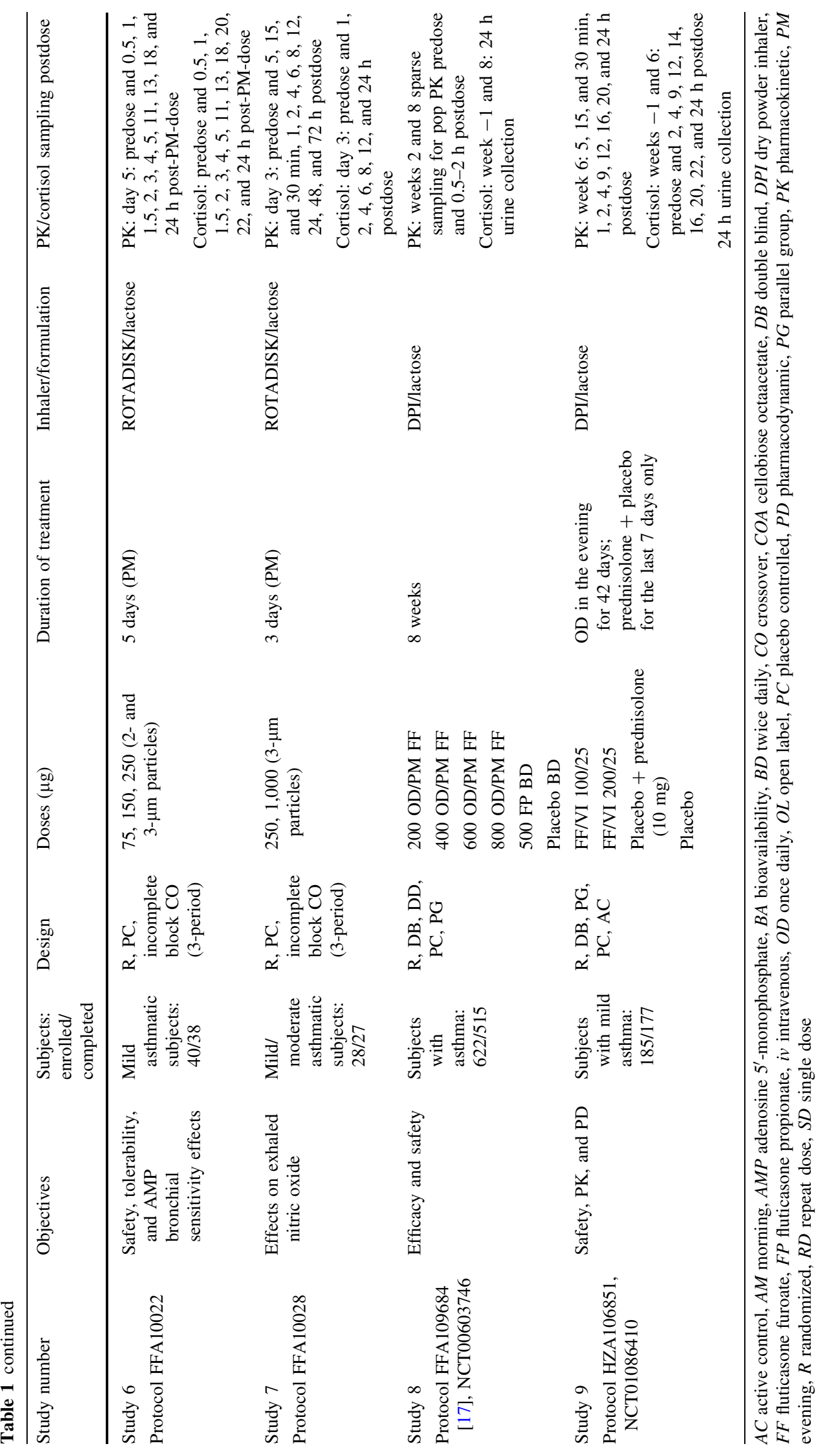


All trials were conducted in compliance with Good Clinical Practice with the ethical principles that have their origins in the Declaration of Helsinki. The investigators obtained Institutional Review Board approvals for the study protocols. All subjects gave their written informed consent before participating in the trial.

Venous blood samples for analysis of plasma fluticasone furoate concentrations were collected in KEDTA tubes at the times detailed in Table 1. The blood samples were put on ice until centrifugation at $1,500 \mathrm{~g}$ for approximately $10 \mathrm{~min}$ at $4{ }^{\circ} \mathrm{C}$. The plasma was transferred into polypropylene containers and frozen at approximately $-20{ }^{\circ} \mathrm{C}$.

\subsection{Bioanalytical Methods}

Plasma samples were analyzed for fluticasone furoate, using either $\left[{ }^{13} \mathrm{C}^{2} \mathrm{H}_{3}\right] \mathrm{GW} 685698$ or $\left[{ }^{13} \mathrm{C}_{3}\right] \mathrm{CCI} 18781$ as internal standard, by solid-phase extraction followed by high-performance liquid chromatography-tandem mass spectrometry (HPLC-MS/MS) using a Perkin Elmer Sciex API 3000. A gradient system using ammonium formate pH 5.0 buffer (26:74, v/v with methanol) and methanol was run with a Phenomex Prodigy ODS3 column $(150 \times 2.0 \mathrm{~mm}$ i.d., $5 \mu \mathrm{m}$ particle size $)$ at $40{ }^{\circ} \mathrm{C}$. The ion transition for fluticasone furoate was $m / z$ 539-313. The validation range of the assay was $10-2,000 \mathrm{pg} / \mathrm{mL}$. Where reported concentrations were above the higher limit of quantification, the plasma samples were diluted, as appropriate, to provide concentrations within the validated range. Interbatch precision (coefficient of variation; CV) was $\leq 8.2 \%$ over the assay range; the lower limit of quantification for fluticasone furoate was $10 \mathrm{pg} / \mathrm{mL}$. Quality controls prepared at three different concentrations were analyzed with each batch of samples against separately prepared calibration standards to assess the day-to-day performance of the assay. Quality control results from this study met the acceptance criteria of no more than one third of the quality control results deviating from the nominal concentration by more than $15 \%$, with at least one quality control result acceptable at each concentration.

\subsection{Pharmacokinetic Analysis}

Fluticasone furoate concentration-time data were subjected to noncompartmental analysis using WinNonlin Pro v2.1 (Pharsight Corporation, Mountain View, CA, USA) or higher to generate estimates of $\mathrm{AUC}_{24}$. The linear trapezoidal rule was used for intervals where the concentration data was increasing and the logarithmic trapezoidal rule was used for intervals where the concentration data was decreasing.

\subsection{Pharmacodynamic Assessments}

\subsubsection{Serum Cortisol Population}

The serum cortisol population consisted of all subjects who did not have protocol violations that were considered to affect the serum cortisol endpoint and whose serum samples were not considered to have confounding factors that would affect the interpretation of the results. Reasons for exclusion from the serum cortisol population included:

- two or more consecutive missing cortisol concentrations over a 24-h collection period. Note: concentrations below the assay's lower limit of quantification were considered nonmissing values for serum cortisol,

- used a protocol-prohibited systemic, oral, or depot corticosteroid during the study,

- used a protocol-prohibited ICS during the study,

- used a protocol-prohibited intranasal corticosteroid during the study,

- used a protocol-prohibited potent cytochrome P450 (CYP) 3A4 inhibitor during the study.

\subsubsection{Urine Cortisol Population}

The urine cortisol population consisted of all subjects who did not have protocol violations that were considered to affect the urine cortisol endpoint and whose urine samples were not considered to have confounding factors that would affect the interpretation of the results. Subjects were excluded from the urine cortisol population prior to breaking the blind. Reasons for exclusion from the urine cortisol population included:

- urine volumes $(0-24 \mathrm{~h})$ of $<600 \mathrm{~mL}$ (women) or $<800 \mathrm{~mL}$ (men),

- 24-h creatinine excretion below the lower limit of threshold range (where the threshold range is defined as the mean \pm 2.5 standard deviations of the observed data),

- collection time intervals outside $24 \pm 2 \mathrm{~h}$,

- used any corticosteroid in violation of the protocol,

- used a protocol-prohibited potent CYP3A4 inhibitor during the study.

The 24-h serum cortisol weighted mean $\left(\mathrm{AUC}_{24} / 24 \mathrm{~h}\right)$ was derived by dividing the AUC (calculated using the linear trapezoidal rule) by the sample collection time interval. The sample collection time interval is defined as the difference between the time of the last cortisol sample and the time of the first cortisol sample. The AUC was calculated from the first nonmissing time points to the last nonmissing time points. Concentrations below the assay's lower limit of quantification were considered nonmissing 
and were set to half the lower limit of quantification when deriving the weighted mean and AUC. If an observation was missing between two nonmissing observations, the AUC was calculated using the measured values at the neighboring time points.

\subsection{Pharmacokinetic/Pharmacodynamic Modeling Procedure}

Population modeling of pharmacokinetic/pharmacodynamic data was performed using nonlinear mixed effect modeling with the computer program NONMEM $^{\circledR}$ v7 (ICON plc, US) running in the predictive modeling environment, a UNIX server-based environment for NON$\mathrm{MEM}^{\circledR}$ analysis. The method selected for minimization was first-order conditional estimation method with interaction [18]. Supporting application interfaces for data handling, exploratory diagnostics, simulation, and graphical representation of the data included Xpose V4 [19], R (The $\mathrm{R}$ Foundation for Statistical Computing Version 2.13.1), PsN, and Excel 2007.

Previous analyses have shown the relationships for fluticasone furoate $\mathrm{AUC}_{24}$ and $\mathrm{WM} 24$ and for fluticasone furoate $\mathrm{AUC}_{24}$ and 24-h urine cortisol excretion to be described by a sigmoid $E_{\max }$ model (GlaxoSmithKline, unpublished data, Study FFA10002 and FFA103096).

Steady-state fluticasone furoate $\mathrm{AUC}_{24}$ values covered a wide range across the studies included in the pharmacokinetic/pharmacodynamic meta-analyses; from noncalculable due to values below the lower limit of quantification (assigned as zero for the analysis) to $>6,300 \mathrm{pg} \cdot \mathrm{h} / \mathrm{mL}$. At the high fluticasone furoate AUC values, some serum cortisol concentrations were reported as nonquantifiable. These nonquantifiable serum cortisol values were set to half the lower limit of quantification for estimation of the WM24. Nonquantifiable urine cortisol concentrations were observed at the high fluticasone furoate AUC values. However, it was not possible to apply an imputation to these records and hence urine cortisol excretion for these cases has been set to missing for the analysis.

Both single-dose and repeat-dose data were available for serum cortisol and therefore a sigmoid $E_{\max }$ model, with different slopes for single and repeat doses, was fitted to fluticasone furoate $\mathrm{AUC}_{24}$ and $\mathrm{WM} 24$ data using NON$\operatorname{MEM}^{\circledR}$ (Eqs. 1, 2).

Effect $=C_{0}-\frac{\left(C_{0}-E_{\max }\right) \times A U C_{24}^{\gamma}}{\left(A U C_{50}^{\gamma}+A U C_{24}^{\gamma}\right)}$

$E_{\text {max }}=\mathrm{C}_{0} \cdot \exp (-k \cdot D a y)$

where $E_{\max }$ is the WM24 value at maximum effect, $C_{0}$ is the WM24 at zero concentration, $\mathrm{AUC}_{50}$ is the $\mathrm{AUC}$ producing $50 \%$ of maximum effect, $\gamma$ is the Hill coefficient, and $k$ is the coefficient on number of days of dosing.

Only repeat-dose data were included for the 24-h urinary cortisol excretion analysis. The following sigmoid $E_{\max }$ model was fitted to fluticasone furoate $\mathrm{AUC}_{24}$ and 24-h urine cortisol data using NONMEM $^{\circledR}$ (Eq. 3).

Effect $=\mathrm{C}_{0}-\frac{\left(\mathrm{C}_{0}-E_{\max }\right) \times A U C_{24}^{\gamma}}{\left(A U C_{50}^{\gamma}+A U C_{24}^{\gamma}\right)}$

where $E_{\max }$ is the urine cortisol at the maximum effect, $C_{0}$ is the urine cortisol at zero concentration, $\mathrm{AUC}_{50}$ is the AUC producing $50 \%$ of maximum effect, and $\gamma$ is the Hill coefficient.

The stepwise covariate model (SCM) building tool of PsN was used to investigate factors that may impact the model parameters, including subject demographic characteristics (sex, age, and weight), formulation/inhaler (fluticasone furoate blended with lactose administered via ROTADISK or administered via DPI, or fluticasone furoate blended with lactose and COA administered via DISKUS/ ACCUHALER), and population (healthy subjects or subjects with asthma). This procedure implements forward selection with criteria of $p=0.05$ followed by backward elimination model selection with criteria of $p=0.01$. Race was to be evaluated, but because $94 \%$ of the serum cortisol population and $85 \%$ of the urine cortisol population were white, it was not deemed appropriate.

Model evaluation to assess the adequacy of the final models, including the effects of statistically significant covariates, was performed using a visual predictive check procedure [20]. This procedure was conducted as follows: 1,000 replicates of the original dataset were simulated, based on the parameter estimates of the final model, and a $95 \%$ prediction interval computed based on the simulated datasets. The observed fluticasone furoate $\mathrm{AUC}_{24}$ versus cortisol data were plotted on the prediction interval to visually assess the concordance between the simulated and observed data.

\section{Results}

\subsection{Subject Demographics}

A summary of subject demographic characteristics for subjects who provided data for the serum cortisol and urine cortisol analyses are presented in Table 2. The majority of data (61.0 and $93.1 \%$, respectively) were provided by subjects with asthma. Data from 372 subjects providing 752 observations were included in the final analysis for serum cortisol, and from 597 subjects providing 682 observations in the final analysis for urine cortisol. 
Table 2 Summary of subject demographic characteristics for the serum cortisol and urine cortisol pharmacokinetic/pharmacodynamic populations

\begin{tabular}{|c|c|c|}
\hline Total number of subjects & Serum cortisol & Urine cortisol \\
\hline Included in meta-analysis, $n$ & 372 & 597 \\
\hline \multicolumn{3}{|l|}{ Population } \\
\hline Healthy subjects, $n(\%)$ & $145(39.0)$ & $41(6.9)$ \\
\hline Subjects with asthma, $n(\%)$ & $227(61.0)$ & $556(93.1)$ \\
\hline \multicolumn{3}{|c|}{ Demographics-total (healthy subjects and asthma) } \\
\hline Age (years), median (range) & $31(12-65)$ & $44(12-75)$ \\
\hline \multicolumn{3}{|l|}{$\operatorname{Sex}, n(\%)$} \\
\hline Female & $88(23.7)$ & $243(40.7)$ \\
\hline Male & $284(76.3)$ & $354(59.3)$ \\
\hline Height (cm), median (range) & $175.0(145-200)$ & $168.0(135-194)$ \\
\hline Weight (kg), median (range) & $\begin{array}{l}79.7 \\
\quad(48.0-125.2)\end{array}$ & $\begin{array}{l}77.0 \\
\quad(41.4-165.0)\end{array}$ \\
\hline \multicolumn{3}{|l|}{ Race, $n(\%)$} \\
\hline $\begin{array}{l}\text { White-White/Caucasian/ } \\
\text { European heritage }\end{array}$ & $350(94)$ & $508(85)$ \\
\hline $\begin{array}{l}\text { African American/African } \\
\text { heritage }\end{array}$ & $12(3)$ & $19(3)$ \\
\hline Asian-East Asian heritage & $1(<1)$ & 0 \\
\hline $\begin{array}{l}\text { Asian-Central/South Asian } \\
\text { heritage }\end{array}$ & $1(<1)$ & $8(1)$ \\
\hline Asian-Japanese heritage & $2(<1)$ & $1(<1)$ \\
\hline Asian-South East Asian heritage & 0 & $18(3)$ \\
\hline American Indian/Native Alaskan & $1(<1)$ & $20(3)$ \\
\hline White-Arabic/North African & $1(<1)$ & $3(<1)$ \\
\hline Other & $4(1)$ & $20(3)$ \\
\hline
\end{tabular}

\subsection{Relationship Between Fluticasone Furoate $\mathrm{AUC}_{24}$ and 24-h Weighted Mean Serum Cortisol}

A sigmoid $E_{\max }$ model, with different slopes for single and repeat doses, was fitted to fluticasone furoate $\mathrm{AUC}_{24}$ and WM24 data using NONMEM ${ }^{\circledR}$. The population parameter estimates for the base model are presented in Table 3. Differences between population and individual estimates were partially explained by interindividual variability on $\mathrm{AUC}_{50}$ and $C_{0}$.

The serum cortisol base model was subjected to SCM building assessing the potential for population type (healthy subjects or subjects with asthma), age, weight, sex, and formulation/inhaler $(\mathrm{FOIH})$ to affect $C_{0}$ or $\mathrm{AUC}_{50}$. The factors of population (healthy subjects or subjects with asthma), age, weight, and sex were not identified as significant covariates from the SCM building. The population parameter estimates for the final model are presented in Table 4. The goodness-of-fit plot for the final model shows that the final model appears to provide a reasonable prediction of the serum cortisol relationship (Fig. 1).
Table 3 Parameter estimates from base model for fluticasone furoate AUC and serum cortisol

\begin{tabular}{lcc}
\hline Parameter & Estimate $(95 \% \mathrm{CI})$ & RSE $(\%)$ \\
\hline$C_{0}(\mathrm{nmol} / \mathrm{L})$ & $215(209-221)$ & 0.304 \\
$\mathrm{AUC}_{50}(\mathrm{pg} \cdot \mathrm{h} / \mathrm{mL})$ & $1,845(1,652-2,060)$ & 0.763 \\
$k$ & $8.08(4.71-13.87)$ & 13.2 \\
$\mathrm{GAM} \mathrm{SD}$ & $0.955(0.767-1.140)$ & 10.0 \\
GAM RD & $3.02(2.29-3.75)$ & 12.4 \\
$C_{0}$ variability & $22.8(19.7-25.3)$ & 12.3 \\
AUC & $54.2(23.9-42.1)$ & 26.2 \\
Proporiabilional error & $15.8(11.8-19.0)$ & 22.3 \\
Additive error $(\mathrm{nmol} / \mathrm{L})$ & $16.5(3.5-23.1)$ & 48.7 \\
\hline
\end{tabular}

$A U C_{50}$ the FF area under the concentration-time curve over $24 \mathrm{~h}$ $\left(\mathrm{AUC}_{24}\right)$ producing $50 \%$ of maximum effect, $C_{O}$ the 24 -h weighted mean at zero $\mathrm{FF} \mathrm{AUC}_{24}, C I$ confidence interval, $F F$ fluticasone furoate, GAM the slope for single dose and repeat dose, $k$ coefficient on the number of days of dosing, $R D$ repeat dose, $R S E$ relative standard error, $S D$ single dose

Table 4 Parameter estimates from final model for fluticasone furoate AUC and serum cortisol

\begin{tabular}{|c|c|c|}
\hline Parameter & Estimate $(95 \% \mathrm{CI})$ & RSE (\%) \\
\hline$C_{0}(\mathrm{nmol} / \mathrm{L})$ & $221(213$ to 230$)$ & 0.404 \\
\hline $\mathrm{AUC}_{50}(\mathrm{pg} \cdot \mathrm{h} / \mathrm{mL})$ & $1,556(1,380$ to 1,755$)$ & 0.829 \\
\hline$k$ & 7.39 (3.42 to 15.96$)$ & 19.7 \\
\hline GAM SD & $0.915(0.648$ to 1.180$)$ & 14.9 \\
\hline GAM RD & 3.03 (2.50 to 3.56$)$ & 8.91 \\
\hline FOIH (DISKUS) on $\mathrm{AUC}_{50}$ & $-0.166(-0.438$ to 0.106$)$ & -83.7 \\
\hline FOIH (DPI) on $\mathrm{AUC}_{50}$ & $0.490(0.255$ to 0.755$)$ & 27.6 \\
\hline FOIH (DISKUS) on $C_{0}$ & $-0.0783(-0.017$ to 0.140$)$ & -40.1 \\
\hline FOIH (DPI) on $C_{0}$ & $0.157(0.070$ to 0.244$)$ & 28.3 \\
\hline$C_{0}$ variability & 21.4 (18.4 to 23.9$)$ & 12.8 \\
\hline $\mathrm{AUC}_{50}$ variability & $27.9(18.4$ to 34.9$)$ & 28.9 \\
\hline Proportional error & 16.0 (11.8 to 19.2$)$ & 23.2 \\
\hline Additive error (nmol/L) & 15.8 (4.11 to 21.98$)$ & 47.6 \\
\hline
\end{tabular}

$A U C_{50}$ the $\mathrm{FF}$ area under the concentration-time curve over $24 \mathrm{~h}$ $\left(\mathrm{AUC}_{24}\right)$ producing $50 \%$ of maximum effect, $C_{O}$ the weighted mean 24 at zero $\mathrm{FF} \mathrm{AUC}_{24}, C I$ confidence interval, DPI dry powder inhaler, $F F$ fluticasone furoate, $F O I H$ formulation/inhaled, GAM slope for single dose and repeat dose, $k$ coefficient on the number of days of dosing, $R D$ repeat dose, $R S E$ relative standard error, $S D$ single dose

Although FOIH was shown to be a significant covariate $(p<0.05)$ on the estimate of $C_{0}$, the median values and ranges for $C_{0}$ for each $\mathrm{FOIH}$ variation were very similar. FOIH was also shown to be a significant covariate on the estimate of $\mathrm{AUC}_{50}$. In the model, ROTADISK provided the greatest number of records $(62 \%)$ and hence was the reference formulation. It should be noted that subjects using DISKUS only constituted $16 \%$ of the overall serum cortisol population. Although the median values of $\mathrm{AUC}_{50}$ were higher for DISKUS and lower for DPI compared with 

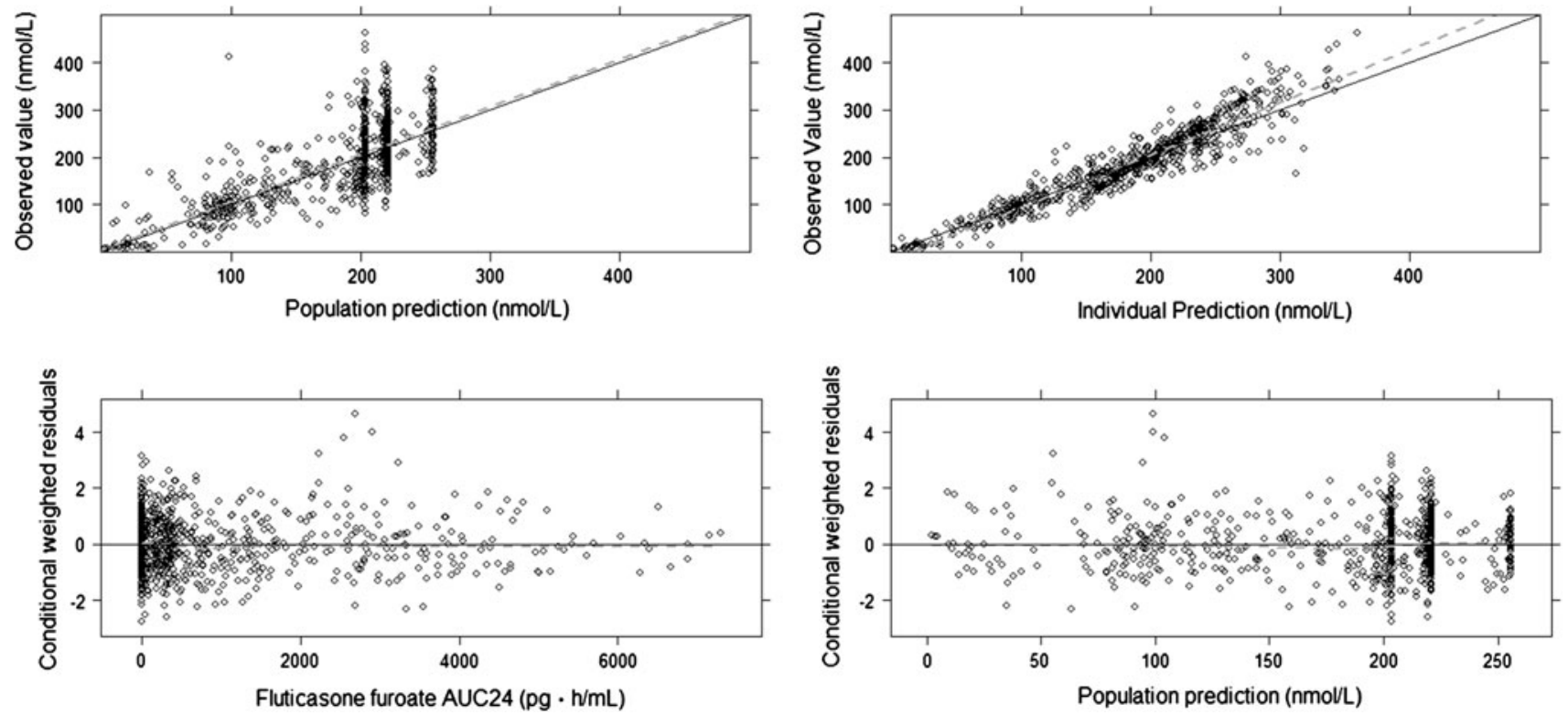

Fig. 1 Diagnostic plots for the final serum cortisol model. Solid lines represent the line of identity or the ordinate value of zero; dashed lines represent a Loess smoother. $A U C_{24}$ area under the concentration-time curve over $24 \mathrm{~h}$ postdose, $F F$ fluticasone furoate

the median $\mathrm{AUC}_{50}$ values for the ROTADISK, the majority of values were within the range of $\mathrm{AUC}_{50}$ values estimated for ROTADISK.

The plot for the visual predictive check for the fluticasone furoate $\mathrm{AUC}_{24}-24$-h serum cortisol model (Fig. 2)

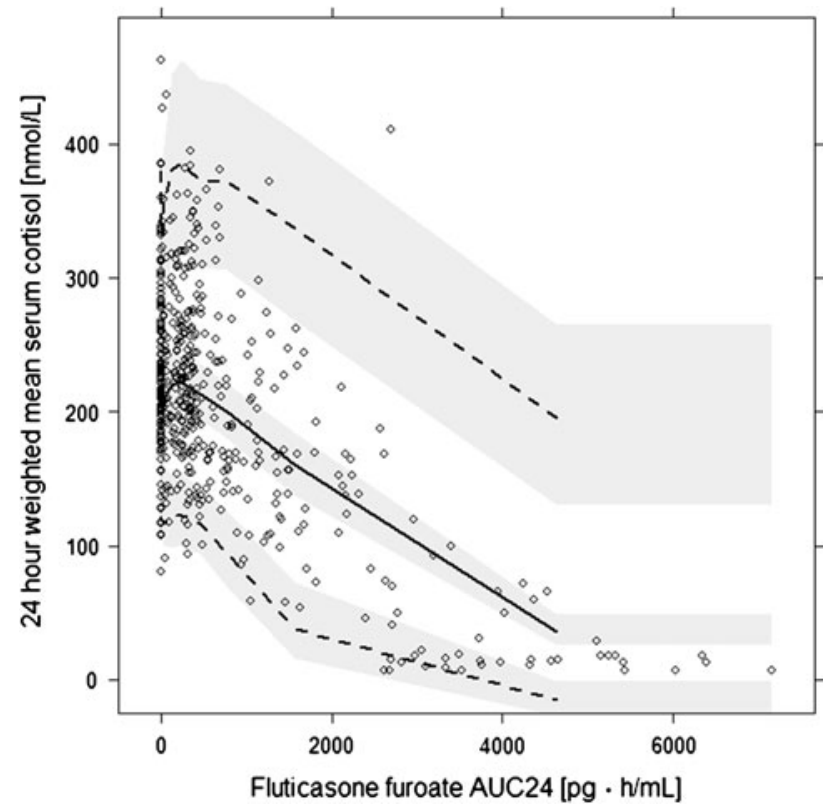

Fig. 2 Visual predictive check for serum cortisol final model. Solid red line is the 50th percentile of the observed data and the dashed lines the 5th and 95th percentiles of the observed data. The shaded areas are the $95 \%$ confidence intervals for the simulated 5th, 50th, and 95 th percentiles. $A U C_{24}$ area under the concentration-time curve over $24 \mathrm{~h}$ postdose, $F F$ fluticasone furoate showed that the majority of the data were captured in the prediction interval encompassing $95 \%$ of the population as indicated by the 2.5 and 97.5 percentile boundaries indicating that the model was valid for this dataset.

\subsection{Relationship Between Fluticasone Furoate $\mathrm{AUC}_{24}$ and 24-h Urinary Cortisol Excretion}

A sigmoid $E_{\max }$ model was also fitted to fluticasone furoate $\mathrm{AUC}_{24}$ and 24-h urine cortisol excretion data using $\mathrm{NONMEM}^{\circledR}$ and the population parameter estimates for the final model are presented in Table 5. Due to the greater variability of the urine cortisol excretion data compared with serum cortisol data it was necessary to fix the slope parameter. Initially the estimate from the serum cortisol model was used (3.06) in the modeling procedure, but covariance could not be obtained with this value, and a value of 3.20 was chosen following evaluation of different estimates.

The urine cortisol base model was subjected to SCM building to assess the potential for population (healthy subjects and subjects with asthma), age, weight, sex, and FOIH to affect $C_{0}$ and $\mathrm{AUC}_{50}$. The factors of population (healthy subjects or subjects with asthma), FOIH, weight, and sex were not significant covariates from the SCM building. The results showed only age to have significant impact on the estimates of both $C_{0}$ and $\mathrm{AUC}_{50}$. Differences between population and individual estimates were partially explained by interindividual variability $(\eta)$ on $\mathrm{AUC}_{50}$ and $C_{0}$. Due to the paucity of data near $E_{\max }$ this parameter was 
Table 5 Parameter estimates from base model for fluticasone furoate AUC and urine cortisol

\begin{tabular}{llc}
\hline Parameter & Estimate $(95 \% \mathrm{CI})$ & $\mathrm{RSE}(\%)$ \\
\hline$C_{0}(\mathrm{mg})$ & $23.1(21.5-24.8)$ & 1.16 \\
$\mathrm{AUC}_{50}(\mathrm{pg} \cdot \mathrm{h} / \mathrm{mL})$ & $1,588(1,380-1,826)$ & 0.963 \\
$E_{\max }(\mathrm{mg})$ & $0.98(-0.549-2.51)$ & 79.6 \\
$\mathrm{GAM}$ & 3.20 fixed & \\
$C_{0}$ variability & $65.3(58.3-71.6)$ & 10.3 \\
$\mathrm{AUC}_{50}$ variability & $40.5(22.8-52.5)$ & 34.8 \\
Proportional error & $26.0(21.7-29.7)$ & 15.6 \\
Additive error $(\mathrm{mg})$ & $2.21(0.443-3.098)$ & 49.0 \\
\hline
\end{tabular}

$A U C_{50}$ the area under the concentration-time curve producing $50 \%$ of maximum effect, $C_{O}$ the $24 \mathrm{~h}$ urine cortisol excretion at zero concentration, $C I$ confidence interval, $E_{\max }$ the 24 urine cortisol excretion at maximum effect, $G A M$ slope for single dose and repeat dose, $R S E$ relative standard error

not well estimated as reflected by the RSE of $58.1 \%$. The population parameter estimates for the final model are presented in Table 6. The goodness-of-fit plots for the final model shows that the final model appears to provide a reasonable prediction of the urine cortisol relationship (Fig. 3).

There was considerable variability in the observed data for 24-h urine cortisol excretion and although age was shown to be a significant covariate based on SCM and objective function there was no obvious improvement in the goodness-of-fit plots or in the estimate of residual variability (Table 5 vs. Table 6).

The plot for the visual predictive check for the fluticasone furoate $\mathrm{AUC}_{24}-24-\mathrm{h}$ urine cortisol model (Fig. 4) showed that the majority of the data were captured within the prediction interval that encompassed $95 \%$ of the population as indicated by the 2.5 and 97.5 percentile boundary indicating that the model was valid for this dataset.

\section{Discussion}

Over a wide range of systemic fluticasone furoate exposure representing the therapeutic and supratherapeutic range, the relationship between fluticasone furoate $\mathrm{AUC}_{24}$ and both WM24 and 24-h urine cortisol excretion was well described by an $E_{\max }$ model. The average estimate of $\mathrm{AUC}_{50}$ was similar for both the serum cortisol and urine cortisol models with values of $1,556 \mathrm{pg} \cdot \mathrm{h} / \mathrm{mL} \quad(95 \%$ CI 1,380-1,755) and 1,686 pg.h/mL (95\% CI 1,480-1,920), respectively. These $\mathrm{AUC}_{50}$ values are notably higher than average fluticasone furoate $\mathrm{AUC}_{24}$ values observed at clinical doses of fluticasone furoate $(\leq 200 \mu \mathrm{g}$; mean $\mathrm{AUC}_{24}$ of $495 \mathrm{pg} \cdot \mathrm{h} / \mathrm{mL}$ for subjects with asthma
Table 6 Parameter estimates from final model for fluticasone furoate AUC and urine cortisol

\begin{tabular}{|c|c|c|}
\hline Parameter & Estimate $(95 \% \mathrm{CI})$ & $\operatorname{RSE}(\%)$ \\
\hline$C_{0}(\mathrm{mg})$ & $22.0(20.5-23.6)$ & 1.13 \\
\hline $\mathrm{AUC}_{50}(\mathrm{pg} \cdot \mathrm{h} / \mathrm{mL})$ & $1,686(1,480-1,920)$ & 0.908 \\
\hline$E_{\max }(\mathrm{mg})$ & $0.707(-0.0990-1.510)$ & 58.1 \\
\hline GAM & 3.20 fixed & \\
\hline Age on $\mathrm{AUC}_{50}$ & $0.0104(0.003-0.018)$ & 35.7 \\
\hline Age on $C_{0}$ & $-0.0141(-0.018$ to -0.010$)$ & -14.3 \\
\hline$C_{0}$ variability & $62.5(51.9-71.6)$ & 15.9 \\
\hline $\mathrm{AUC}_{50}$ variability & $42.5(29.3-52.5)$ & 26.9 \\
\hline Proportional error & $25.7(18.7-31.1)$ & 24.1 \\
\hline Additive error (mg) & $2.03(0.825-2.746)$ & 42.6 \\
\hline
\end{tabular}

$A U C_{50}$ the area under the concentration-time curve producing $50 \%$ of maximum effect, $C_{O}$ the $24 \mathrm{~h}$ urine cortisol excretion at zero concentration, $C I$ confidence interval, $E_{\max }$ the $24 \mathrm{~h}$ urine cortisol at maximum effect, GAM slope for single dose and repeat dose, $R S E$ relative standard error

administered fluticasone furoate/vilanterol 200/25 $\mu \mathrm{g}$; GlaxoSmithKline unpublished data, 2012). Based on the models, a fluticasone furoate $\mathrm{AUC}_{24}$ of $1,000 \mathrm{pg} \cdot \mathrm{h} / \mathrm{mL}$ would be required to reduce 24-h serum cortisol or 24-h urine cortisol excretion by 20 and $17 \%$, respectively. For the serum $E_{\max }$ model, there were different slopes for the single-dose and repeat-dose data. From the results of this model, it can be seen that the same fluticasone furoate $\mathrm{AUC}_{24}$ obtained following repeat dosing results in a greater suppression of serum cortisol, compared with the level of suppression observed after a single exposure. This is an expected finding because serum cortisol measured after a single dose includes cortisol produced prior to dosing and does not reflect the true magnitude of cortisol suppression produced under steady-state conditions. Maximum effect can be observed after 3-4 days of dosing once fluticasone furoate steady state is achieved. Although the median values and ranges for $C_{0}$ for each FOIH were very similar, FOIH was shown to be a significant covariate on the estimates of $C_{0}$. This may be a spurious finding because $C_{0}$ is the cortisol level at zero concentration, and hence impact by FOIH is implausible. Therefore, this finding is likely just a reflection of study-to-study variability. FOIH was also shown to be a significant covariate on the estimate of $\mathrm{AUC}_{50}$. As with $C_{0}$, this finding may also be a reflection of study-to-study variability. Also of note, for studies used in the serum cortisol analysis, ROTADISK and DISKUS administration included doses up to ten times and eight times greater than the maximum fluticasone furoate therapeutic dose of $200 \mu \mathrm{g}$, whilst for DPI the maximum dose was equivalent. Hence, for DPI the full effect on serum cortisol would not have been observed and may have impacted the estimate of $\mathrm{AUC}_{50}$. 

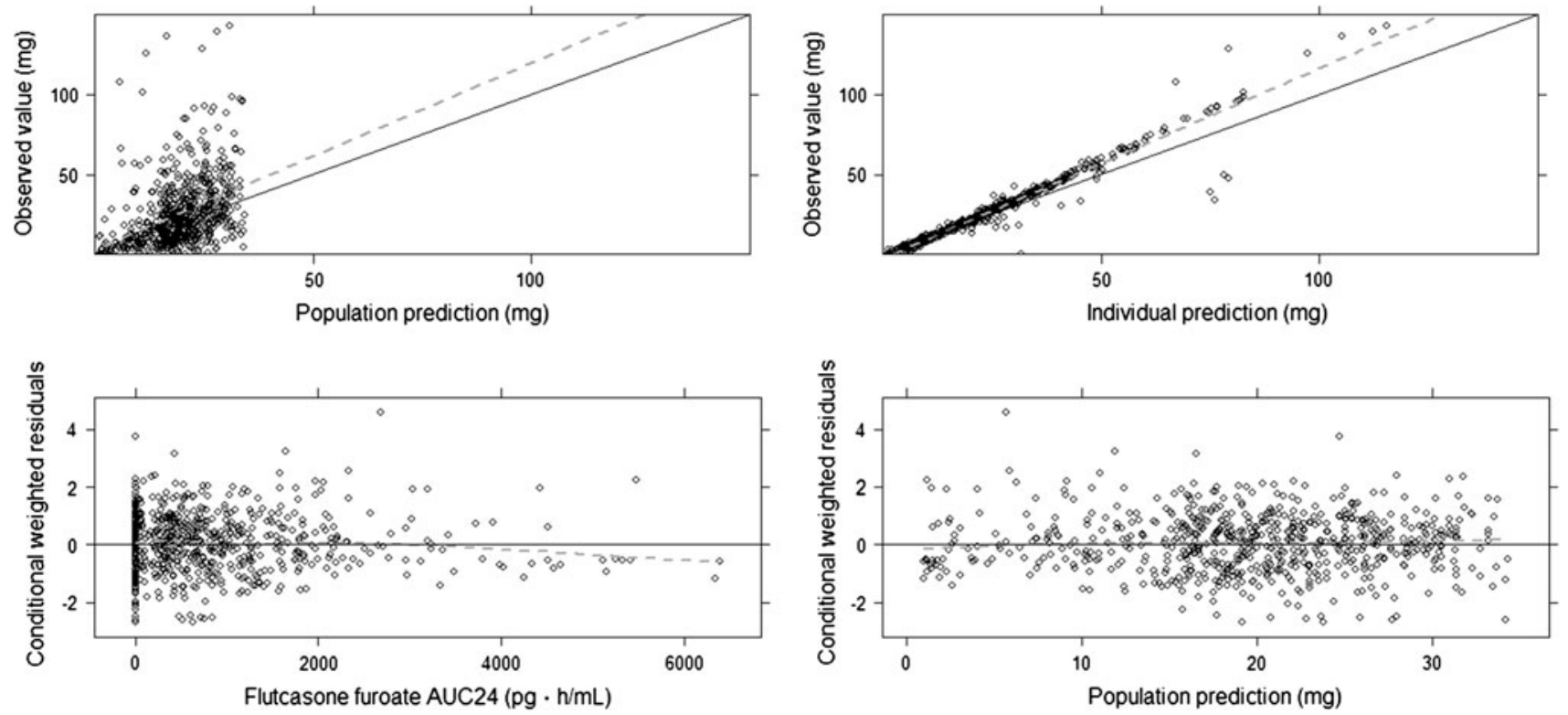

Fig. 3 Diagnostic plots for the final urine cortisol model. Solid lines represent the line of identity or the ordinate value of zero; dashed lines represent a Loess smoother. $A U C_{24}$ area under the concentration-time curve over $24 \mathrm{~h}$ postdose, $F F$ fluticasone furoate

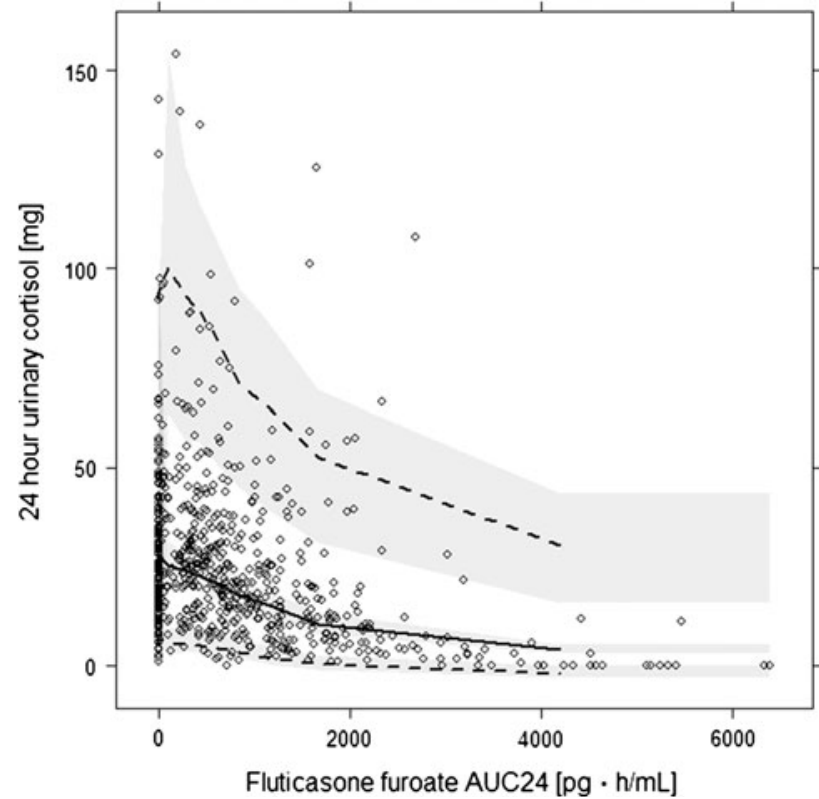

Fig. 4 Visual predictive check for urine cortisol final model. Solid red line is the 50th percentile of the observed data and dashed lines are the 5th and 95th percentiles of the observed data. The shaded areas are the $95 \%$ confidence interval for the simulated 5th, 50th, and 95 th percentiles. $A U C_{24}$ area under the concentration-time curve over $24 \mathrm{~h}$ postdose, $F F$ fluticasone furoate

For the relationship between fluticasone furoate $\mathrm{AUC}_{24}$ and 24-h urine cortisol excretion, age was shown to be a significant covariate on the estimates of both $C_{0}$ and $\mathrm{AUC}_{50}$. There was considerable variability in the observed data for 24-h urine cortisol excretion, and although age was shown to be a significant covariate based on SCM and objective function there was no obvious improvement in the goodness-of-fit plots or in the estimate of residual variability. This finding may in part be explained by the lower underlying 24-h urine cortisol excretion in adolescent subjects compared with adults reported in the literature [21]. In addition, age was not identified as a significant covariate for the serum cortisol model and therefore the relevance of age as a covariate on estimate of $C_{0}$ and $\mathrm{AUC}_{50}$ for the urine cortisol relationship is not clear and is considered to be a reflection of lower urine cortisol excretion in adolescents.

Although the analysis included data from a number of studies, the analytical methodology for fluticasone furoate analysis in plasma was very similar, sensitive, and selective, and included robust internal assay validation. This was also the case for the analytical methods used for both serum and urine cortisol where sensitive and selective HPLC-MS assays were used for all studies. Therefore, combining data from these studies for the pharmacokinetic/ pharmacodynamic analysis is not considered to have any impact on the outcome.

Similar relationships have been described for other ICS, notably an $E_{\max }$ model has been described for fluticasone propionate [22], which estimated an $\mathrm{AUC}_{50}$ of approximately 2,000 $\mathrm{pg} \cdot \mathrm{h} / \mathrm{mL}$ (after adjustment for the difference due the inadequate selectivity of the radioimmunoassay used to analyze samples for fluticasone propionate in that study compared with the more selective HPLC-MS assay [23]). This would indicate that fluticasone furoate is 1.25 times more potent on reduction in cortisol compared with 
fluticasone propionate. However, to put into context of relative therapeutic index, it should be noted that the daily clinical dose of fluticasone furoate $(100$ or $200 \mu \mathrm{g})$ is five times lower than that of fluticasone propionate (500 or $1,000 \mu \mathrm{g}$ ), and, therefore, the therapeutic index for fluticasone furoate with respect to cortisol reduction is no worse than that for fluticasone propionate.

\section{Conclusion}

A pharmacokinetic/pharmacodynamic model has been established over a wide range of systemic fluticasone furoate exposure representing the therapeutic and supratherapeutic range to both 24-h weighted WM24 and 24-h urine cortisol excretion. The values of $\mathrm{AUC}_{50}$ of 1,556 and $1,686 \mathrm{pg} \cdot \mathrm{h} / \mathrm{mL}$ are notably higher than average fluticasone furoate $\mathrm{AUC}_{24}$ values observed at clinical doses of fluticasone furoate $(\leq 200 \mu \mathrm{g})$. Based on the models, a fluticasone furoate $\mathrm{AUC}_{24}$ of $1,000 \mathrm{pg} \cdot \mathrm{h} / \mathrm{mL}$ would be required to reduce 24-h serum cortisol or 24-h urine cortisol excretion by 20 and $17 \%$, respectively.

Acknowledgments The author meets the criteria for authorship set forth by the International Committee for Medical Journal Editors, was involved in the preparation of the article and approved the final draft for submission. The author thanks the subjects and staff who participated in each of the studies. Bioanalytical support for the fluticasone furoate analyses was provided by Worldwide Bioanalysis, Ware, Herts, UK. Editorial support in the form of copyediting and graphic services was provided by Ian Grieve at Gardiner-Caldwell Communications and was funded by GlaxoSmithKline. All the studies used in this analysis were funded by GlaxoSmithKline.

Statement of interest A.A. is employed by and holds stock in GlaxoSmithKline.

Role of the Funding Source This study was funded by GlaxoSmithKline and the sponsor was involved in the design and conduct of the studies, collection of data, and analysis and interpretation of the data. The author had access to all of the data, was involved in every stage of the preparation of the article, performed the statistical analysis, and approved the final version. The sponsor did not place any restriction on authors about the statements made in the final article.

Open Access This article is distributed under the terms of the Creative Commons Attribution Noncommercial License which permits any noncommercial use, distribution, and reproduction in any medium, provided the original author(s) and the source are credited.

\section{References}

1. Lötvall J, Bakke P, Bjermer L, et al. Safety and efficacy of fluticasone furoate/vilanterol trifenatate (FF/VI) in COPD patients. Eur Respir J 2010;24:1013s.

2. Kempsford R, Allen A, Bareille P, et al. The safety, tolerability, pharmacodynamics and pharmacokinetics of inhaled fluticasone furoate (FF) and vilanterol (VI) are unaffected by administration in combination. Eur Respir J. 2011;38(Suppl. 55):138s.

3. Lötvall J, Bakke PS, Bjermer L, et al. Efficacy and safety of 4 weeks' treatment with combined fluticasone furoate/vilanterol in a single inhaler given once daily in COPD: a placebo-controlled randomised trial. BMJ Open. 2012;2:e000370.

4. Busse WW, O'Byrne PM, Bleecker ER, et al. Safety and tolerability of the novel inhaled corticosteroid (ICS) fluticasone furoate (FF) in combination with the long-acting beta 2 agonist (LABA) vilanterol (VI) administered once daily (OD) in patients with asthma. Eur Respir J. 2012;40(Suppl. 56):P2092.

5. Boscia JA, Pudi KK, Zvarich MT, et al. Effect of fluticasone furoate (FF)/vilanterol (VI) administered once daily on $24 \mathrm{~h}$ pulmonary function in patients with COPD: a randomized, threeway, incomplete block, cross-over study. Am J Resp Crit Care Med. 2012;185(Meeting abstracts):A2939.

6. Agusti A, De Backer W, De Teresa L, et al. Efficacy of combination fluticasone furoate/vilanterol (FF/VI) and salmeterol/fluticasone propionate (SFC) over 12 weeks in patients with COPD. Eur Respir J. 2012;40(Suppl. 56):P2889.

7. Bleecker ER, Lötvall J, O'Bryne PM, et al. Efficacy of fluticasone furoate $(\mathrm{FF})$ as a monotherapy and in combination with vilanterol (VI) over 12 weeks in patients with persistent asthma. Eur Respir J. 2012;40(Suppl. 56):P2091.

8. O'Byrne PM, Bleecker ER, Bateman ED, et al. Efficacy and safety of fluticasone furoate (FF)/vilanterol (VI) once daily (OD) for 24 weeks in persistent asthma. Eur Respir J. 2012;40(Suppl. 56):P1794.

9. Lipworth BJ, Seckl JR. Measures for detecting systemic bioactivity with inhaled and intranasal corticosteroids. Thorax. 1997;52:476-82.

10. Lipworth BJ. Systemic adverse effects of inhaled corticosteroid therapy: a systemic review and meta-analysis. Arch Intern Med. 1999;159:941-55.

11. Bernstein DI, Allen DB. Evaluation of tests of hypothalamicpituitary-adrenal axis function used to measure effects of inhaled corticosteroids. Ann Allergy Asthma Immunol. 2007;98:118-27.

12. Masoli M, Weatherall M, Holt S, et al. Inhaled fluticasone propionate and adrenal effects in adult asthma: systematic review and meta-analysis. Eur Respir J. 2006;28:960-7.

13. Clark DJ, Grove A, Cargill RI, et al. Comparative adrenal suppression with inhaled budesonide and fluticasone propionate in adult asthmatic patients. Thorax. 1996;51:262-6.

14. Bruni FM, De Luca G, Venturoli V, et al. Intranasal corticosteroids and adrenal suppression. NeuroImmunoModulation. 2009; $16: 353-62$.

15. Crowley S. Inhaled glucocorticoids and adrenal function: an update. Paediatr Respir Rev. 2003;4:153-61.

16. Bleecker ER, Bateman ED, Busse WW, et al. Consistently favorable safety profile of fluticasone furoate (FF), a once-daily (OD) inhaled corticosteroid (ICS), across a range of treatment steps in patients with uncontrolled asthma. AJRCCM. 2011;183: A1300 (Abstract).

17. Busse WW, Bleecker ER, Bateman ED, et al. Fluticasone furoate demonstrates efficacy in patients with asthma symptomatic on medium doses of inhaled corticosteroid therapy: an 8-week, randomised, placebo-controlled trial. Thorax. 2012;67:35-41.

18. Beal SL, Sheiner LB. NONMEM users guides, Version 5. NONMEM Project Group, University of California, San Francisco. 1996.

19. Jonsson EN, Karlsson MO. Xpose-an S-PLUS based population pharmacokinetic/pharmacodynamic model building aid for NONMEM. Comput Methods Programs Biomed. 1999;58:51-64.

20. Post TM, Freijer JI, Ploeger BA, et al. Extensions to the visual predictive check to facilitate model performance evaluation. J Pharmacokinet Pharmacodyn. 2008;35:185-202. 
21. Juselius RE, Kenny FM. Urinary free cortisol excretion during growth and aging: correlation with cortisol production rate and 17-hydroxycorticosteroid excretion. Metabolism. 1974;23:847-52.

22. Mackie AE, Bye A. The relationship between systemic exposure to fluticasone propionate and cortisol reduction in healthy male volunteers. Clin Pharmacokinet. 2000;39(Suppl. 1):47-54.
23. Daley-Yates PT, Tournant J, Kunka RL. Comparison of the systemic availability of fluticasone propionate in healthy volunteers and patients with asthma. Clin Pharmacokinet. 2000;39 (Suppl. 1):39-45. 